\title{
Hyperostosing sphenoid wing meningiomas: surgical outcomes and strategy for bone resection and multidisciplinary orbital reconstruction
}

\author{
Cecilia L. Dalle Ore, MD, ${ }^{1}$ Stephen T. Magill, MD, PhD, ${ }^{1}$ Roberto Rodriguez Rubio, MD, ${ }^{1}$ \\ Maryam N. Shahin, MD, ${ }^{1}$ Manish K. Aghi, MD, PhD, ${ }^{1}$ Philip V. Theodosopoulos, MD, ${ }^{1}$ \\ Javier E. Villanueva-Meyer, MD, ${ }^{2}$ Robert C. Kersten, MD, ${ }^{3}$ Oluwatobi O. Idowu, MD, ${ }^{3}$ \\ M. Reza Vagefi, MD, ${ }^{3}$ and Michael W. McDermott, MD' \\ Departments of ${ }^{1}$ Neurological Surgery, ${ }^{2}$ Radiology, and ${ }^{3}$ Ophthalmology, University of California, San Francisco, California
}

OBJECTIVE Hyperostosing sphenoid wing meningiomas cause bony hyperostosis that may extend into the orbit, resulting in proptosis, restriction of extraocular movements, and/or compressive optic neuropathy. The extent of bony removal necessary and the optimal reconstruction strategy to prevent enophthalmos is debated. Herein, the authors present their surgical outcomes and reconstruction results.

METHODS This is a retrospective review of 54 consecutive patients undergoing resection of sphenoid wing meningiomas associated with bony hyperostosis. The majority of cases were operated on by the senior author. Extent of tumor resection, volumetric bone resection, radiographic exophthalmos index, complications, and recurrence were analyzed.

RESULTS The median age of the cohort was 52.1 years, with women comprising $83 \%$ of patients. Proptosis was a presenting symptom in $74 \%$, and $52 \%$ had decreased visual acuity. The WHO grade was I (85\%) or II (15\%). The median follow-up was 2.6 years. On volumetric analysis, a median $86 \%$ of hyperostotic bone was resected. Gross-total resection of the intracranial tumor was achieved in $43 \%$ and the orbital tumor in $27 \%$, and of all intracranial and orbital components in $20 \%$. Orbital reconstruction was performed in $96 \%$ of patients. Postoperative vision was stable or improved in $98 \%$ of patients and diplopia improved in $89 \%$. Postoperative complications occurred in $44 \%$ of patients, and $26 \%$ of patients underwent additional surgery for complication management. The most frequent complications were medical complications and extraocular movement deficits. The median preoperative exophthalmos index was 1.26, which improved to $1.12 \mathrm{im}-$ mediately postoperatively and to 1.09 at the 6 -month follow-up $(p<0.001)$. Postoperatively, 18 patients $(33 \%)$ underwent adjuvant radiotherapy after subtotal resection. Tumors recurred/progressed in 12 patients (22\%).

CONCLUSIONS Resection of hyperostosing sphenoid wing meningiomas, particularly achieving gross-total resection of hyperostotic bone with a good aesthetic result, is challenging and associated with notable medical and ocular morbidity. Recurrence rates in this series are higher than previously reported. Nevertheless, the authors were able to attain improvement in proptosis and visual symptoms in the majority of patients by using a multidisciplinary approach.

https://thejns.org/doi/abs/10.3171/2019.12.JNS192543

KEYWORDS hyperostosis; meningioma; sphenoorbital; proptosis; reconstruction; exophthalmos index; oncology

$\mathrm{S}$ PHENOID wing meningiomas represent $14 \%-18 \%$ of all intracranial meningiomas. ${ }^{17,29}$ They are accompanied by bony hyperostosis in approximately $40 \%$ of cases, ${ }^{30}$ particularly in en plaque or sphenoorbital meningiomas. ${ }^{32,35}$ Bony hyperostosis is usually larger than the intradural tumor mass ${ }^{1,6,12}$ and is secondary to tumor infiltration of bone and stimulation of osteoblast activity. ${ }^{6,35}$ Frequent sites of hyperostosis include the sphenoid wing, lateral and superior orbit, and anterior clinoid..$^{35}$ The multicompartmental nature of the tumor makes complete removal challenging, and rates of residual disease are reported up to $27 \%{ }^{30,35}$ Bony hyperostosis and the intradural tumor can compress cranial nerves $(\mathrm{CNs})$ and cause proptosis, visual deterioration, and cosmetic deformity. ${ }^{21,35}$ On histopathological review, more than $90 \%$ of hyperostotic bone is infiltrated by viable meningioma cells. ${ }^{23} \mathrm{In}$

ABBREVIATIONS CN = cranial nerve; EI = exophthalmos index; EOM = extraocular muscle; EOR = extent of resection; GTR = gross-total resection; HSWM = hyperostosing sphenoid wing meningioma; STR = subtotal resection.

SUBMITTED September 15, 2019. ACCEPTED December 16, 2019.

INCLUDE WHEN CITING Published online March 6, 2020; DOI: 10.3171/2019.12.JNS192543. 
addition to bone infiltration, hyperostosing sphenoid wing meningiomas (HSWMs) can infiltrate the extraconal orbit, periorbita/intraconal space, ${ }^{34}$ and temporalis muscle. ${ }^{8,23}$, 34,35 The majority of patients with HSWM present with symptomatic proptosis, ${ }^{6,12,29,30,34,35}$ and restoration of neutral globe position, without exophthalmos or enophthalmos, is one of the primary surgical objectives. Moreover, the extent of hyperostotic bone resection is inconsistently reported and rarely quantified in studies of HSWM surgical outcomes.

There is controversy in the surgical treatment of these tumors-including the goal for extent of resection (EOR), necessity of orbital reconstruction, and adjuvant radiotherapy. ${ }^{12,14,18,35}$ Surgical approach and aggressiveness of resection is often guided by the patient's presenting symptoms, including proptosis, decreased visual acuity from compressive optic neuropathy or exposure keratopathy, and/or extraocular muscle (EOM) dysfunction. Thus, we performed a retrospective review of a single institution's experience treating HSWM that is notable for volumetric analysis of the extent of hyperostotic bone resection and the use of a multidisciplinary approach to orbital reconstruction.

\section{Methods}

A retrospective review of consecutive patients who underwent resection of sphenoid wing meningioma at our institution was performed. Inclusion criteria were age older than 18 years and patients who underwent resection of an HSWM with adequate records available. Preoperative imaging was reviewed for all patients to identify those with sphenoid wing meningiomas having a component of bony hyperostosis. Patients who did not undergo surgical treatment or who did not have hyperostosis were excluded. The Committee on Human Research at our institution approved this study.

\section{Surgical Technique}

The patient's head is rotated $20^{\circ}$ with the tumor side up. Intraoperative neuromonitoring was used early in the series but has since been discontinued because it did not influence our resection. A coronal incision is used to avoid excessive tension on the scalp flap and allow intraoperative assessment of orbit position relative to the contralateral, normal side. The eyes are protected with ophthalmic ointment, and a temporary tarsorrhaphy is performed. The drapes are placed at the level of the midmaxilla to keep the eyes in the surgical field. The scalp flap is elevated separately from the pericranium, which is incised along the superior temporal line bilaterally and reflected forward. A temporalis interfascial dissection releases the scalp forward and protects the frontalis nerve. The temporalis muscle is released from its bony attachments and reflected inferiorly and posteriorly.

Image guidance with a CT bone window is used to delineate tumor-involved bone. A frontotemporal craniotomy is performed, followed by removal of the tumor-involved bone. The tumor-involved bone is removed down to the meningoorbital band and lateral floor of the middle cranial fossa. The periorbita is dissected from the orbital roof and lateral wall to the anterior limit of tumor-involved bone, and an orbitozygomatic osteotomy including the zygomatic arch is performed. The dura mater is reflected off the floor of the middle fossa to identify the foramen rotundum, spinosum, and ovale, in this respective order. Tumor-involved bone is removed as necessary, respecting predefined limits for safe resection, as shown in Fig. 1. Once the tumorinvolved bone is removed, the dura is opened over the sphenoid wing with a C-shaped incision, and the intradural tumor is resected along with the dura down to the level of the superior orbital fissure. The dura is reconstructed with autologous pericranium that is sutured in place and augmented with fibrin glue. Resection is then transitioned to the oculoplastic surgeon who performs en bloc removal of tumor-involved periorbita posteriorly until the superior orbital fissure is reached, separating it from the frontal nerve superiorly and lateral rectus muscle temporally.

\section{Orbital Reconstruction}

The periorbital defect is reconstructed by placing an onlay collagen sponge over the exposed defect. The roof and lateral wall are reconstructed with titanium mesh-embedded porous polyethylene implant (Medpor Titan Cranial Curve; Stryker). Early in our experience, we used autologous bone from the calvarial inner table or bare titanium mesh. ${ }^{19}$ The implant is screwed to the posterior aspect of the roof of the orbit on the orbitozygomatic osteotomy and then tapered to fit by using wire-cutting scissors (Figs. 2 and 3). After a satisfactory reconstruction is performed and the orbitozygomatic bone piece is secured, the scalp flap is reflected back over the craniotomy site to expose the eyes. The tarsorrhaphy is released, and the globe position on each side is compared. The orbital implant can be revised as needed to achieve symmetrical globe position. Once this is complete, the oculoplastic surgeon performs a forced duction test to ensure no entrapment of the EOMs by the orbital implant. ${ }^{13}$ The pterional bone flap is secured and convexity defects are repaired with multilayer titanium mesh and closed in standard fashion. ${ }^{15}$ Postoperatively, patients received 7 days of antiepileptic therapy, typically with levetiracetam or phenytoin.

\section{Data Collection}

Demographic data and baseline characteristics were collected, including age, sex, preoperative symptoms, prior treatment, and, if available, formal preoperative ophthalmological examination. Operative variables included preoperative embolization, description of orbital reconstruction, and postoperative complications. Postoperative variables included reoperation, adjuvant radiotherapy, disease recurrence, and time to recurrence.

For patients with pre- and postoperative CT imaging, the bony hyperostosis volume was measured in order to allow for calculation of extent of bony resection. The EOR was determined radiographically and described for both dural and orbital components separately. Near-total resection was classified as subtotal resection (STR). The preoperative and postoperative exophthalmos indexes (EIs) were measured from the CT or T1-weighted MRI; the EI is the ratio of the distance from the anterior aspect of the 

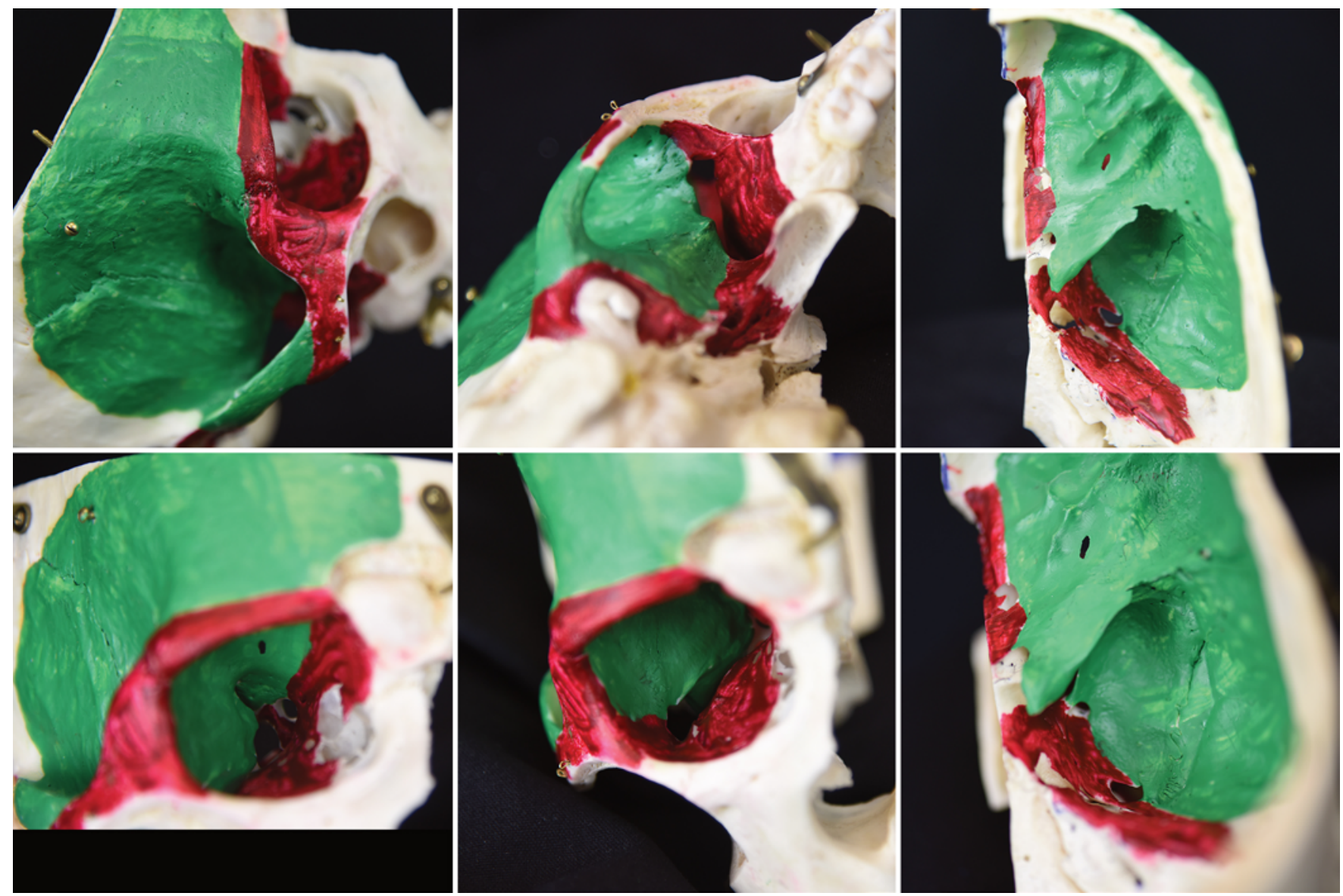

FIG. 1. Safe zones for bony resection. Green-painted regions of the skull represent safe regions for bony resection including the superior and lateral orbital walls, pterion, zygoma, and lateral middle fossa floor. Red-painted structures that limit resection include the orbital rim; medial and inferior orbital walls; sphenoid and ethmoid sinus walls; intraconal orbit; petrous and sphenoid bone medial to foramen rotundum, ovale, and spinosum; and posterior petrous bone around the temporomandibular joint. These regions are left unresected to preserve $\mathrm{CN}$ and jaw function as well as optimize cosmetic appearance. Figure is available in color online only.

globe of each eye to a line connecting the tips of the frontal processes of the zygoma. ${ }^{4}$ Postoperative EI was measured both in the immediate postoperative period (within 2 weeks of surgery) and at 6 months postoperatively. Normal EI was defined as 0.9-1.1, with an EI $<0.9$ defined as enophthalmos and an EI $>1.1$ defined as exophthalmos. Tumor-involved bone was digitized on the pre- and postoperative CT. To determine bony EOR, the bone considered safe for resection (green-painted regions in Fig. 1) was quantified and compared to the bone remaining on the postoperative CT scan.

Paired Student t-tests were used to compare preoperative EI to postoperative EI in immediate postoperative imaging and 6-month follow-up imaging. Prevalence of exophthalmos was compared using McNemar's test for paired binary variables. Kaplan-Meier survival analysis with log-rank tests was used to compare time to recurrence by EOR.

\section{Results}

In total, 54 patients with HSWM were identified. Demographics and presenting symptoms are shown in Table
1. Preoperative visual acuity was decreased in 28 patients (52\%), nearly all of which was due to compressive optic neuropathy, with only 1 patient having exposure keratopathy. Eleven patients (20\%) had abnormalities in EOM function on examination. The median length of follow-up was 2.6 years.

The pathology was WHO grade I $(\mathrm{n}=46,85 \%)$ and WHO grade II $(\mathrm{n}=8,15 \%)$. The meningioma extended into the orbit in 46 patients (85\%). Preoperative embolization was performed in 7 patients (13\%). Orbital reconstruction was performed in 52 patients (96\%; Table 2). Three methods were used for reconstruction (Figs. 2 and 3 )-a modified titanium mesh-embedded porous polyethylene implant $(\mathrm{n}=35,65 \%)$, bone graft $(\mathrm{n}=13,24 \%)$, or titanium mesh $(\mathrm{n}=4,7 \%)$.

Preoperative and postoperative CT scans were available for 24 patients (44\%) to conduct analysis of the bony volume of resection. The median preoperative hyperostotic bone volume was $23.0 \mathrm{~cm}^{3}$ (range $4.3-91.2 \mathrm{~cm}^{3}$ ) and the median postoperative volume was $3.3 \mathrm{~cm}^{3}$ (range $0.6-10.9$ $\left.\mathrm{cm}^{3}, \mathrm{p}<0.001\right)$. Volumetric analysis of hyperostotic bone resection was conducted in all 24 patients with pre- and postoperative CT scans. Only 2 patients (8\%) achieved 

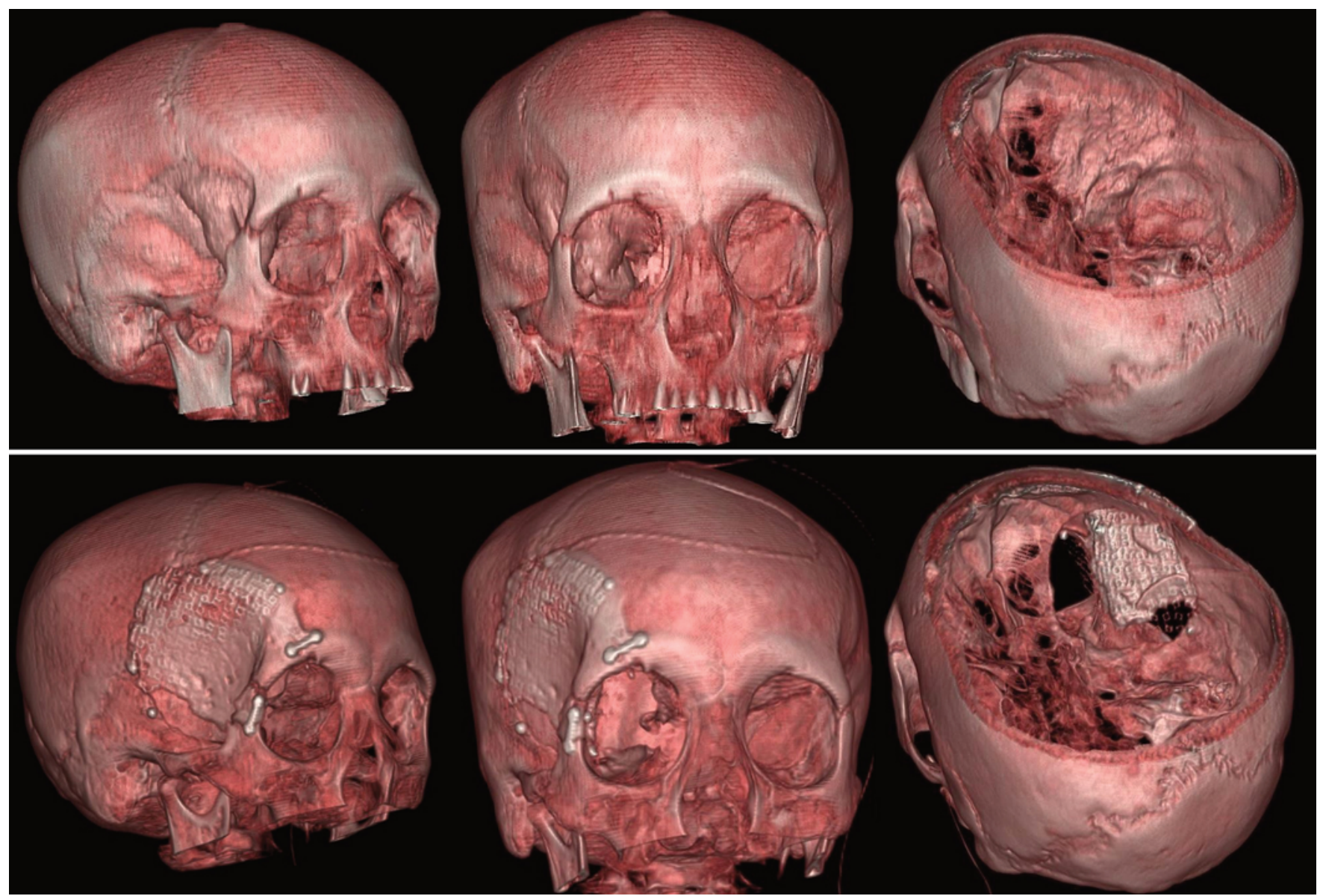

FIG. 2. 3D reconstructions of pre- (upper row) and postoperative (lower row) CT scan sequences. This patient had an HSWM that underwent reconstruction with a polyethylene implant for orbital reconstruction and titanium mesh to repair the calvarial defect, the method currently used by the senior author (M.W.M.). Figure is available in color online only.

gross-total resection (GTR) of hyperostotic bone (data not shown). The median percentage of hyperostotic bone resected was $86 \%$ (range 59\%-97\%). Intracranial EOR was available for 49 patients and was subtotal in $28(57 \%)$ and gross total in $21(43 \%)$. Of the 47 patients with intraorbital invasion, the extent of intraorbital resection was available for 45 patients and was subtotal in $33(73 \%)$ and gross total in $12(27 \%)$.

Of 28 patients with reduced visual acuity preoperatively, 12 (43\%) improved. Overall, visual acuity was improved in 12 patients (22\%), stable in 41 patients (76\%), and worse in 1 patient (2\%). Of 11 patients with EOM dysfunction preoperatively, 6 (55\%) improved. Overall, EOM function was improved in 6 patients $(11 \%)$, stable in 42 patients (78\%), and worse in 6 patients (11\%).

Overall, 18 patients (33\%) underwent adjuvant radiation therapy postoperatively. Meningiomas progressed postoperatively in $22 \%$ of all patients, at a median 2.8 years. Progression occurred in the orbit $(\mathrm{n}=8)$, orbit and cavernous sinus $(\mathrm{n}=1)$, lateral wall of the cavernous sinus $(\mathrm{n}=1)$, floor of the middle fossa $(\mathrm{n}=1)$, and infratemporal fossa $(\mathrm{n}=1)$. In 11 of 12 cases, progression occurred following STR of that compartment; 1 patient with intraorbital GTR and intracranial STR developed progression and re- currence at the cavernous sinus, middle fossa floor, and posterolateral orbit. Of the 12 patients who experienced disease progression, $2(17 \%)$ underwent repeat surgery and radiation, 2 (17\%) underwent repeat surgery alone, and $7(58 \%)$ received radiation therapy alone. One patient elected to continue observation. The extent of hyperostotic bone resection (OR 1.4, 95\% CI 0.1-33.0, $\mathrm{p}=0.341$ ), intracranial tumor resection (OR 3.0, 95\% CI 0.6-15.6, $\mathrm{p}$ $=0.310)$, and orbital resection (OR 1.0, 95\% CI 0.2-4.3, $\mathrm{p}=0.259$ ) were not significantly associated with disease progression.

Postoperative complications occurred in 24 patients (44\%; Table 3). Medical complications included seizures $(\mathrm{n}=2,4 \%)$, arrhythmia $(\mathrm{n}=2,4 \%)$, acute on chronic kidney disease $(n=1,2 \%)$, pulmonary embolism $(n=1,2 \%)$, and alcohol withdrawal $(\mathrm{n}=1,2 \%)$. The most common surgical complications were new neurological deficit and infection. Transient neurological deficits occurred in 5 patients (9\%), and included aphasia responsive to steroids in 2 patients, new EOM dysfunction in 2 patients, and reduced visual acuity in 1 patient. Permanent new neurological deficits occurred in 7 patients $(13 \%)$ and included new EOM dysfunction in 6 patients $(11 \%)$ and reduced visual acuity in 1 patient (2\%). One patient had formation of an 


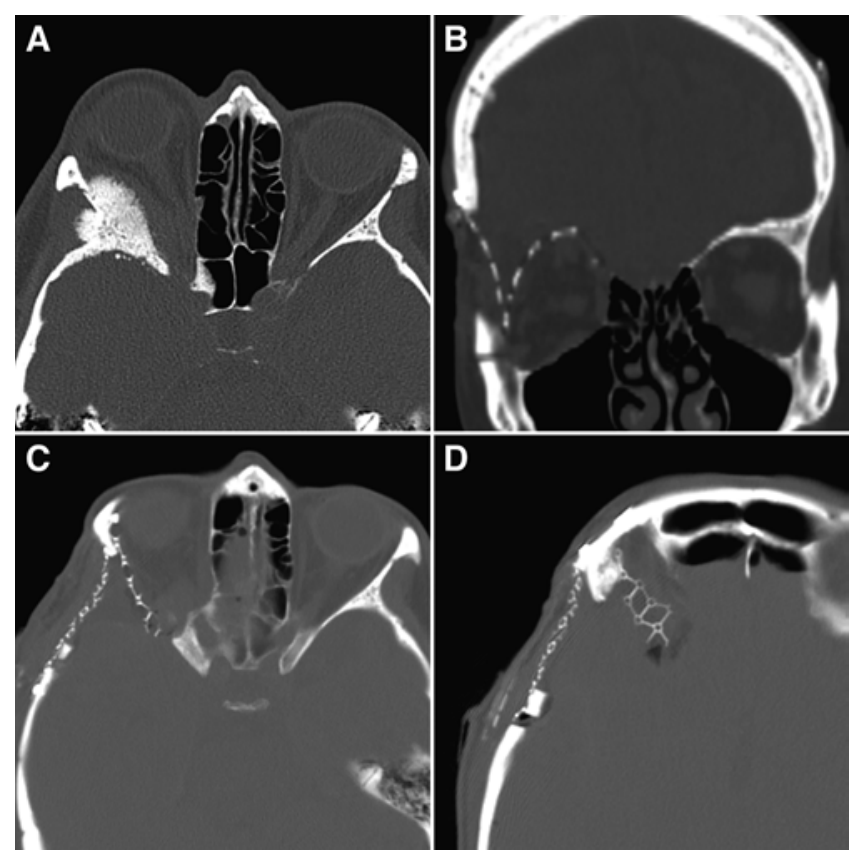

FIG. 3. CT scans showing reconstruction with a titanium mesh-embedded porous polyethylene implant. A: Preoperative axial CT scan showing bony hyperostosis of a right-sided HSWM. B-D: Coronal (B) and axial (C and D) CT images demonstrating orbital reconstruction with the titanium mesh-embedded porous polyethylene plate.

intraorbital hematoma during preoperative neuromonitoring electrode placement in the superior aspect of the orbit that punctured the superior ophthalmic vein. As a result of an orbital compartment syndrome, a lateral canthotomy with cantholysis was required and the surgery was aborted prior to incision. Wound infections occurred in 3 patients $(6 \%)$ and wound breakdown without evidence of infection

TABLE 1. Demographics and preoperative characteristics in 54 patients with HSWMs

\begin{tabular}{lc}
\hline \multicolumn{1}{c}{ Characteristic } & Value \\
\hline Sex & \\
\hline Male & $9(17 \%)$ \\
\hline Female & $45(83 \%)$ \\
\hline Median age, yrs (range) & $52.1(30.6-79.5)$ \\
\hline Prior surgery & $4(7 \%)$ \\
\hline Presenting symptom & \\
\hline Proptosis & $40(74 \%)$ \\
\hline Headache & $22(41 \%)$ \\
\hline Palpable abnormality & $5(9 \%)$ \\
\hline Seizure & $4(7 \%)$ \\
\hline Incidental & $3(6 \%)$ \\
\hline Preop ophthalmological status & \\
\hline Decreased visual acuity & $28(52 \%)$ \\
\hline EOM abnormality & $11(20 \%)$ \\
\hline
\end{tabular}

Values are presented as the number of patients (\%) unless otherwise indicated.
TABLE 2. Tumor characteristics and surgical outcomes

\begin{tabular}{|c|c|}
\hline Variable & Value \\
\hline Non-bony tumor w/in orbit & $46(85 \%)$ \\
\hline \multicolumn{2}{|l|}{ WHO grade } \\
\hline I & $46(85 \%)$ \\
\hline II & $8(15 \%)$ \\
\hline \multicolumn{2}{|l|}{ Hyperostotic bone volumetrics, $n=24$} \\
\hline Median preop vol, $\mathrm{cm}^{3}$ (range) & $23.0(4.3-91.2)$ \\
\hline Median residual vol, $\mathrm{cm}^{3}$ (range) & $3.3(0.6-10.9)$ \\
\hline Median EOR, \% (range) & $85.7(59.4-96.6)$ \\
\hline \multicolumn{2}{|l|}{ Intracranial EOR, $n=49$} \\
\hline STR & $28(57 \%)$ \\
\hline GTR & $21(43 \%)$ \\
\hline \multicolumn{2}{|l|}{ Orbital EOR, $n=45$} \\
\hline STR & $33(73 \%)$ \\
\hline GTR & $12(27 \%)$ \\
\hline \multicolumn{2}{|l|}{ Intracranial \& intraorbital EOR, $n=45$} \\
\hline STR & $36(80 \%)$ \\
\hline GTR & $9(20 \%)$ \\
\hline \multicolumn{2}{|l|}{ Orbital reconstruction } \\
\hline Polyethylene implant, Medpor & $35(65 \%)$ \\
\hline Autologous bone graft, calvarial inner table & $13(24 \%)$ \\
\hline Mesh & $4(7 \%)$ \\
\hline None & $2(4 \%)$ \\
\hline \multicolumn{2}{|l|}{ Visual \& EOM outcomes } \\
\hline \multicolumn{2}{|l|}{ Follow-up visual acuity } \\
\hline Improved & $12(22 \%)$ \\
\hline Stable & $41(76 \%)$ \\
\hline Worse & $1(2 \%)$ \\
\hline \multicolumn{2}{|l|}{ Preop visual deficit, $n=28$} \\
\hline Improved & $12(43 \%)$ \\
\hline Stable & $16(57 \%)$ \\
\hline Worse & $0(0 \%)$ \\
\hline \multicolumn{2}{|l|}{ Follow-up EOM function } \\
\hline Improved & $6(11 \%)$ \\
\hline Stable & $42(78 \%)$ \\
\hline Worse & $6(11 \%)$ \\
\hline \multicolumn{2}{|l|}{ Preop EOM dysfunction, $n=11$} \\
\hline Improved & $6(55 \%)$ \\
\hline Stable & $5(45 \%)$ \\
\hline Worse & $0(0 \%)$ \\
\hline Adjuvant postop XRT after STR & $18(33 \%)$ \\
\hline \multicolumn{2}{|l|}{ Progression } \\
\hline Disease progression & $12(22 \%)$ \\
\hline Median time to progression, yrs (range) & $2.8(0.4-4.8)$ \\
\hline \multicolumn{2}{|l|}{ Treatment after radiographic progression } \\
\hline Continued observation & $1(8 \%)$ \\
\hline Reop for progression & $2(17 \%)$ \\
\hline XRT for progression & $7(58 \%)$ \\
\hline Reop \& XRT for progression & $2(17 \%)$ \\
\hline
\end{tabular}

$\mathrm{XRT}=$ radiation therapy.

Values are presented as the number of patients (\%) unless otherwise indicated. 
TABLE 3. Complications and management of HSWMs

\begin{tabular}{|c|c|}
\hline Complication & №. $(\%)$ \\
\hline Postop complications & $24(44 \%)$ \\
\hline Medical complications & $7(13 \%)$ \\
\hline Seizure & $2(4 \%)$ \\
\hline Arrhythmia & $2(4 \%)$ \\
\hline AKI & $1(2 \%)$ \\
\hline PE & $1(2 \%)$ \\
\hline Alcohol withdrawal & $1(2 \%)$ \\
\hline Surgical complications & $19(35 \%)$ \\
\hline Aborted surgery & $1(2 \%)$ \\
\hline \multicolumn{2}{|l|}{ Neurological deficit } \\
\hline Transient & $5(9 \%)$ \\
\hline Aphasia & $2(4 \%)$ \\
\hline EOM & $2(4 \%)$ \\
\hline Visual acuity & $1(2 \%)$ \\
\hline Permanent & $7(13 \%)$ \\
\hline EOM & $6(11 \%)$ \\
\hline Visual acuity & $1(2 \%)$ \\
\hline Orbital entrapment & $2(4 \%)$ \\
\hline Wound infection/breakdown requiring reop & $4(7 \%)$ \\
\hline $\begin{array}{l}\text { Tension pneumocephalus requiring needle } \\
\text { decompression }\end{array}$ & $1(2 \%)$ \\
\hline Pseudomeningocele (resolved w/ cap) & $1(2 \%)$ \\
\hline Reops & $14(26 \%)$ \\
\hline Plastic surgery reop & $3(6 \%)$ \\
\hline Fat graft for temporal hollowing & $2(4 \%)$ \\
\hline Reshaping for contour defect & $1(2 \%)$ \\
\hline Washout/wound revision & $4(7 \%)$ \\
\hline Ophthalmological reop & $7(13 \%)$ \\
\hline Blepharoptosis & $3(6 \%)$ \\
\hline Entrapment & $2(4 \%)$ \\
\hline Strabismus & $2(4 \%)$ \\
\hline Exotropia & $1(2 \%)$ \\
\hline Biopsy orbital contents & $1(2 \%)$ \\
\hline
\end{tabular}

$\mathrm{AKI}=$ acute kidney injury; $\mathrm{PE}=$ pulmonary embolism.

Values are presented as the number of patients (\%) unless otherwise indicated.

occurred in 1 patient (2\%), all of whom returned to the operating room.

Two patients who had undergone reconstruction with a polyethylene orbital implant required a washout; no infection of the periorbita was noted in either patient and neither orbital implant was removed. The bone flap was removed in 1 patient, followed by cranioplasty after completion of antibiotics. A third patient had undergone orbital reconstruction with an autologous bone graft, which was left in place, and a methylmethacrylate cranioplasty, which was removed. One patient developed tension pneumocephalus requiring needle decompression, and 1 patient developed a pseudomeningocele that resolved with a compressive cap. Two patients developed restrictive strabismus postoperatively due to lateral rectus entrapment by the implant, requiring a return to the operating room with oculoplastic surgery for implant repositioning via a lateral orbitotomy. One reoperation for entrapment was prior to forced ductions, and the second occurred following the introduction of forced ductions. In the case that occurred with intraoperative forced duction, limited adduction secondary to orbital fat entrapment between the implant and the lateral orbit was noted during the index surgery and released with improvement in duction. Postoperatively, the patient was noted to have persistent restriction in adduction. On return to the operating room, entrapment of the lateral rectus by both the lateral and posteroinferior components of the implant was noted and addressed, with complete resolution.

Overall, reoperation for complications occurred in 14 patients (26\%). In total, 7 patients (13\%) underwent reoperation with oculoplastic surgery. Surgery was performed for blepharoptosis $(n=3,6 \%)$, entrapment $(n=2,4 \%)$, restrictive strabismus $(\mathrm{n}=2,4 \%)$, exotropia $(\mathrm{n}=1,2 \%)$, and biopsy of orbital contents $(n=1,2 \%)$. Several of the 7 patients had multiple ophthalmological symptoms present. Three patients (6\%) underwent reoperation with plastic surgery to improve the aesthetic outcome; of these, 2 patients received fat grafting for temporal hollowing, and 1 patient underwent recontouring of a bony defect.

The median preoperative EI was 1.26 (range 0.98-1.73; Table 4), and $83 \%$ of patients had exophthalmos on imaging preoperatively. An illustrative case showing how the EI was calculated is shown in Fig. 4. Immediately postoperatively, the median EI was 1.12 (range 0.91-1.40, p < 0.001), with a median change of -0.10 (range -0.6 to 0.40 ), and $53.7 \%$ of patients had exophthalmos $(n=29, p<0.001)$. At 6 months postoperatively, the median EI was 1.09 (range $0.90-1.30, \mathrm{p}<0.001)$, with a median change of -0.17 , and 19 patients $(35.2 \%, \mathrm{p}<0.001)$ had exophthalmos.

\section{Discussion}

HSWMs are surgically challenging tumors. The goals of resection are optimizing tumor control via maximizing the EOR of tumor in all compartments (dura, bone, and orbit), minimizing morbidity, and treating presenting symptoms. In this series, resolution of exophthalmos was achieved in a majority of patients by the 6-month followup. However, this was associated with significant morbid-

TABLE 4. Exophthalmos index in 54 patients with HSWMs

\begin{tabular}{lccccc}
\hline & Preop & Immediate Postop & $\mathrm{p}$ Value & 6 Mos Postop & $p$ Value \\
\hline Median El (range) & $1.26(0.98$ to 1.73$)$ & $1.12(0.91$ to 1.40$)$ & $<0.001$ & $1.09(0.90$ to 1.30$)$ & $<0.001$ \\
\hline Change in El (range) & & $-0.10(-0.6$ to 0.4$)$ & & $-0.17(-0.68$ to 0.6$)$ & \\
\hline Exophthalmos, $\mathrm{n}=54$ & $45(83.3 \%)$ & $29(53.7 \%)$ & $<0.001$ & $19(35.2 \%)$ & $<0.001$ \\
\hline
\end{tabular}




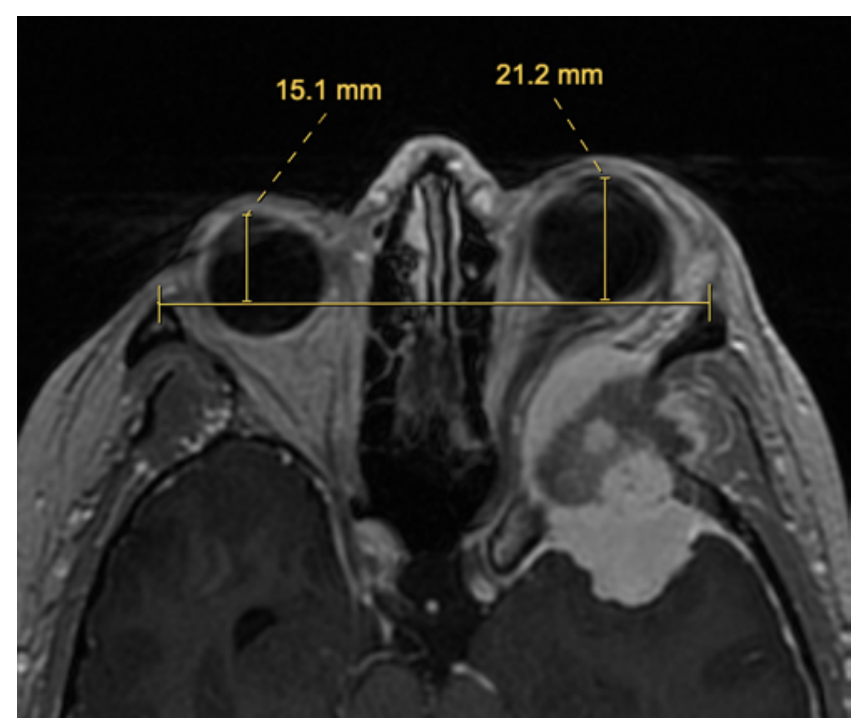

FIG. 4. Illustrative case of HSWM causing proptosis and measurement of El. Axial T1-weighted postcontrast MR image showing a left-sided HSWM causing proptosis. The El is calculated by dividing the affected side over the nonaffected side $(21.2 / 15.1=1.40)$. Figure is available in color online only.

ity, with $26 \%$ of patients requiring additional surgery to treat postoperative complications. Furthermore, nearly all patients underwent STR of meningioma in at least one compartment; GTR of all bony hyperostotic components was particularly challenging to achieve due to extension into the sphenoid bone medial to $\mathrm{CN}$ foramina, posteriorly into the petrous bone around the temporomandibular joint, and anteriorly into the apical and medial orbit. This residual tumor probably contributed to a $20 \%$ rate of progression/recurrence.

\section{EOR and Strategy for Bone Resection}

Radiographic GTR of all intracranial components was achieved in only $43 \%$ of patients, and GTR of bony components was achieved in only $8 \%$ of patients. The literature indicates that the intradural tumor is often amenable to GTR, ${ }^{19,29,34,35}$ but complete removal of all intracranial tumor is very challenging, with residual tumor in $11 \%-100 \%$ of cases. ${ }^{30,35}$

The EOR is often determined by tumor anatomy: softtissue structures that limit resection include involvement of the cavernous sinus, superior orbital fissure and Zinn's fibrous ring, intraconal orbit, pterygopalatine fossa, and vasculature. $6,29,30,34,35$ The necessary EOR of hyperostotic bone is controversial; whereas some authors advocate for maximal safe removal of all involved bone, ${ }^{3,4,8,29}$ others have prioritized only complete dural resection with removal of grossly tumor-infiltrated, "pockmarked"-appearing bone. ${ }^{6,34,35}$ Resection of the lateral orbital wall has been suggested to treat proptosis, but this is accompanied with the potential for enophthalmos..$^{27,28,34}$

Our practice has been to maximize the extent of safe resection of all components. We have defined the perceived limits for safe bony resection (green areas in Fig. 1) and the areas that should not be removed due to increased risk for complications (red areas in Fig. 1). For the medial orbit, the safe limit of bone resection is along the shared wall with the anterior and posterior ethmoid air cells. Posteriorly, the roof and lateral wall of the optic canal and clinoid process can be safely removed. Involved bone between the superior and inferior orbital fissures can be removed. In the floor of the middle fossa, tumor in the greater sphenoid wing lateral to the foramen rotundum, ovale, and spinosum can be removed safely. The roof of the temporal mandibular joint fossa is not removed, and it defines the posterior and lateral limit along the floor of the middle fossa.

Volumetric analysis of hyperostosis resection is not typically reported in the literature. We have found that a significant amount of hyperostotic bone may remain, even after removing large amounts of bone. Given the evidence that hyperostotic bone contains meningioma cells in addition to reactive bone, ${ }^{23}$ volumetric analysis of hyperostotic bone resection may allow for improved understanding of the total extent of tumor resection and corresponding oncological outcomes, with implications for postoperative imaging surveillance.

\section{Reconstruction of the Orbit}

The aims of reconstruction after HSWM resection include minimizing CSF leakage, prevention of infection, symmetrical eye position, prevention of globe pulsation, and acceptable frontotemporal scalp cosmesis. ${ }^{4,29}$ Reconstruction of the temporal bone defect can be performed using titanium mesh, ${ }^{35}$ methylmethacrylate, ${ }^{6,34,35}$ hydroxyapatite, ${ }^{10}$ or split bone autografts. ${ }^{29,35}$

Whereas reconstruction of the orbital rim is always necessary, ${ }^{23}$ reconstruction of the orbit is controversial. Historically, orbital reconstruction has been recommended if more than one wall of the orbit is resected. $8,10,12,16,27,29,33$ Some authors have advocated for orbital reconstruction if only the medial or lateral walls are resected..$^{23,31}$ However, others have argued that orbital reconstruction is not necessary at all.,6,7,14,18,20,21,24,30,34,35 Talacchi et al. attributed a case of pulsatile exophthalmos in 1 patient who did not undergo orbital reconstruction to inadequate dural suspension of the frontal lobe. ${ }^{34}$ Likewise, authors who typically do not advocate for reconstruction suggest that it may be necessary if the periorbita is incised, with resultant loss of the supportive integrity of the soft tissue of the orbit. ${ }^{7,34,35}$ Nevertheless, reported complication rates are similar in cases with and without orbital reconstruction. 2,8,12,24,27,30

Overall, we found that reconstruction of the orbit using an implant in collaboration with an oculoplastic surgeon allows for excellent postoperative positioning of the globe. Such a multidisciplinary approach allows for aggressive tumor resection with reduction of proptosis, while safeguarding the function of the eye. We prefer the polyethylene implant because it can be molded and shaped easily. Forced duction testing may reduce the incidence of orbital entrapment, although incomplete release of entrapped orbital components following a positive forced duction test may result in persistent entrapment requiring reoperation. No patients required removal of the orbital implant for infection. Overall, we believe that the benefits of reconstruction exceed the potential for reconstruction-associated complications. 


\section{Symptomatic Management}

Proptosis is the most frequent presenting symptom, occurring in $75 \%-96 \%$ of patients, ${ }^{6,12,29,30,34,35}$ with higher rates of exophthalmos evident on imaging. ${ }^{30}$ Although rates of improvement in symptomatic proptosis are as high as $60 \%-100 \%,, 21,23,30,35$ residual proptosis remains in 9\%$76 \%$ of cases. ${ }^{8,11,12,21,23,24,27,30,34}$ Symptomatic enophthalmos may also occur in $2 \%-7 \%$ of patients. ${ }^{12,35}$ In this series, $74 \%$ of patients reported symptomatic proptosis preoperatively and $25 \%$ noted symptomatic residual proptosis, which was mild and decreased in all but 2 patients. Enophthalmos occurred in 1 patient. The EI improved from a median preoperative score of 1.26 to 1.09 at the 6-month follow-up, consistent with the literature. .,10,30 $^{-1}$

Reduction in visual acuity is frequently present in HSWMs. Reduced visual acuity was observed preoperatively in $51.9 \%$ of patients in this series, consistent with the rate of $20 \%-52 \%$ in the literature. ${ }^{4,12,29,30,34,35}$ Visual field deficits are less common, occurring in 3\%-36\%.,12,30 $\mathrm{Al}$ though rates of visual acuity improvement are lower than rates of proptosis improvement, recovery of vision following surgery can and does occur. Improvement in visual acuity is reported in $45 \%-100 \%$ of patients with preoperative visual symptoms, ${ }^{4,6,8,23,30,34}$ with complete resolution in $21 \%-57 \%$, consistent with our results. ${ }^{30,34,35}$ However, transient or permanent visual acuity loss has been reported in $20 \%$ and $7 \%-12 \%$, respectively, which is higher than our $2 \% .^{12,35}$ Decompression of the optic canal, if involved, may increase the blood supply to the optic nerve and raise the chances of visual stabilization or improvement. ${ }^{9,35}$ Similarly, diplopia due to EOM dysfunction has been reported in $6 \%-38 \%, 4,12,29,34$ which improves postoperatively in 50\%$89 \%$ of patients. ${ }^{23,34}$ Transient and permanent postoperative EOM dysfunction was reported in $7 \%$ and $13 \%-16 \%$, respectively. ${ }^{12,35}$ The $11 \%$ observed in this series is similar to those and represents the majority of new postoperative neurological deficits.

\section{Complications and Reoperations}

Within this cohort, postoperative neurological deficits, including worsening of visual acuity or EOM function, were a major complication of HSWM resection. In addition to a permanent reduction in visual acuity and EOM function, transient neurological deficits were observed in 9.3\% of patients in this series, including aphasia and EOM dysfunction. Rates of neurological deficits are reported as high as $11 \%-60 \%$ for transient neurological deficits and $12.5 \%-30 \%$ for permanent neurological deficits. ${ }^{29,30,34}$ Neurological deficits most frequently involve CN III, ${ }^{29,30,34}$ although CN VII and V deficits, hemiplegia, and decreased visual acuity are also reported. ${ }^{4,29,30}$

The $44 \%$ rate of postoperative complications is consistent with the literature and reflects the significant morbidity associated with resection. ${ }^{12}$ Reported complications include CSF leakage, ${ }^{34,35}$ seizure, ${ }^{35}$ infection, ${ }^{29,34,35}$ hematoma requiring a return to the operating room, ${ }^{29,34}$ and stroke., ${ }^{4,29}$, 30,35 Strategies we implemented to reduce perioperative complications include placement of a lumbar drain to prevent CSF leakage (used in 16 of 54 patients in this series) and forced duction testing to identify restrictive strabismus. Overall, $26 \%$ of our patients returned to the operat- ing room for complication management. Aesthetics may motivate some reoperations. Temporal hollowing is common, occurring in 33\%-100\% of patients. ${ }^{12,35}$ Although patients are often not concerned, ${ }^{12}$ some may undergo further intervention to improve cosmesis, as in our series. ${ }^{35}$

\section{Progression and Adjuvant Therapy}

In this cohort, $33 \%$ of patients underwent adjuvant radiotherapy for residual disease. Some have advocated for adjuvant radiotherapy, particularly following STR,, 48,23 whereas others recommend against it, especially when the pathology is WHO grade I. ${ }^{12,35}$ Based on recent data from prospective phase 2 trials in Europe and North America, and our own studies of recurrence, we offer upfront adjuvant radiotherapy for WHO grade II tumors or those in which the MIB labeling index is $>7 \%$ or residual WHO grade I tumors with demonstrated growth. ${ }^{5,25,26,36}$ Concerns regarding radiotherapy include optic neuropathy, ${ }^{12,35}$ cataracts, EOM dysfunction, ${ }^{4}$ reduced feasibility of reoperation in an irradiated field, ${ }^{12,35}$ and pituitary insufficiency; ${ }^{4}$ however, these risks are smaller than the risks of reoperation.

HSWMs have a higher recurrence and progression rate than comparable meningiomas in other locations. ${ }^{35} \mathrm{We}$ observed a $22 \%$ rate of disease progression at a median follow-up of 2.6 years, consistent with the reported range of $10 \%-56 \% .4,29,30,34,35$ Terrier et al. reported that there was no difference in the recurrence rate between total and subtotal removal. ${ }^{35}$ Although we observed no difference in progression rate by EOR, a relatively low number of patients (20\%) underwent GTR, particularly of both bone and orbital components. Residual hyperostotic bone may contribute to the high rates of recurrence observed and is independent of the extent of dural resection. ${ }^{18}$ HSWMs frequently involve structures such as the cavernous sinus, where recurrence is attributed to limited resection. ${ }^{18,21,29}$ Improved reporting of the extent of hyperostotic bone resection may aid in characterization of recurrence and progression patterns in these tumors.

\section{Limitations of the Study}

This is a retrospective cohort, single-institution study, and it is not possible to determine causation with regard to time to recurrence from our data. The median followup time available for our patients was short in the context of the natural history of meningiomas, ${ }^{22}$ so the recurrence rate is probably lower than it would be if follow-up for 1015 years were available. Additionally, availability of data regarding EOR was inconsistent, particularly with regard to Simpson grade, which was not available for a majority of patients and was therefore omitted from the analysis. Volumetric bone analysis was only available for $44 \%$ of patients because not all patients had postoperative CT scans. Nevertheless, we think this is an important metric to follow and now routinely collect these data.

\section{Conclusions}

Resection of HSWMs is challenging, and GTR of all hyperostotic bone is rarely possible. Resection of hyperostotic bone of the superior, lateral, and posterior orbital walls and intraorbital tumor with concomitant orbital re- 
construction can produce significant improvements in the EI and symptomatic proptosis, but it is associated with morbidity. When reconstruction of the orbit is performed, intraoperative examination of eye position and forced duction testing should be performed before closure to identify EOM entrapment. We have found that involvement of an oculoplastic surgeon in the pre-, intra-, and postoperative management of these patients is helpful in achieving the best possible outcomes.

\section{Acknowledgments}

This work was supported in part by the Linda Wolfe Memorial Meningioma Research Project.

\section{References}

1. Arana E, Diaz C, Latorre FF, Menor F, Revert A, Beltrán A, et al: Primary intraosseous meningiomas. Acta Radiol 37:937-942, 1996

2. Bikmaz K, Mrak R, Al-Mefty O: Management of bone-invasive, hyperostotic sphenoid wing meningiomas. J Neurosurg 107:905-912, 2007

3. Bonnal J, Thibaut A, Brotchi J, Born J: Invading meningiomas of the sphenoid ridge. J Neurosurg 53:587-599, 1980

4. Bowers CA, Sorour M, Patel BC, Couldwell WT: Outcomes after surgical treatment of meningioma-associated proptosis. J Neurosurg 125:544-550, 2016

5. Chen WC, Magill ST, Wu A, Vasudevan HN, Morin O, Aghi MK, et al: Histopathological features predictive of local control of atypical meningioma after surgery and adjuvant radiotherapy. J Neurosurg 130:443-450, 2018

6. De Jesús O, Toledo MM: Surgical management of meningioma en plaque of the sphenoid ridge. Surg Neurol 55:265269, 2001

7. DeMonte F, Tabrizi P, Culpepper SA, Suki D, Soparkar CN, Patrinely JR: Ophthalmological outcome after orbital entry during anterior and anterolateral skull base surgery. J Neurosurg 97:851-856, 2002

8. Gaillard S, Pellerin P, Dhellemmes P, Pertuzon B, Lejeune JP, Christiaens JL: Strategy of craniofacial reconstruction after resection of spheno-orbital "en plaque" meningiomas. Plast Reconstr Surg 100:1113-1120, 1997

9. Han SJ, Magill ST, Tarapore PE, Horton JC, McDermott MW: Direct visualization of improved optic nerve pial vascular supply following tuberculum meningioma resection: case report. J Neurosurg 125:565-569, 2016

10. Heller RS, David CA, Heilman CB: Orbital reconstruction for tumor-associated proptosis: quantitative analysis of postoperative orbital volume and final eye position. J Neurosurg [epub ahead of print March 8, 2019; DOI: 10.3171/2018.12. JNS181385]

11. Heufelder MJ, Sterker I, Trantakis C, Schneider JP, Meixensberger J, Hemprich A, et al: Reconstructive and ophthalmologic outcomes following resection of spheno-orbital meningiomas. Ophthal Plast Reconstr Surg 25:223-226, 2009

12. Honeybul S, Neil-Dwyer G, Lang DA, Evans BT, Ellison DW: Sphenoid wing meningioma en plaque: a clinical review. Acta Neurochir (Wien) 143:749-758, 2001

13. Idowu OO, Kalin-Hajdu E, Grumbine FL, Kersten RC, McDermott M, Vagefi MR: Restrictive strabismus following frontotemporal-orbitozygomatic craniotomy. Cureus 9:e1937, 2017

14. Kiyofuji S, Casabella AM, Graffeo CS, Perry A, Garrity JA, Link MJ: Sphenoorbital meningioma: a unique skull base tumor. Surgical technique and results. J Neurosurg [epub ahead of print August 23, 2019; DOI: 10.3171/2019.6.JNS191158]
15. Lau D, McDermott MW: A method for combining thin and thick malleable titanium mesh in the repair of cranial defects. Cureus 7:e267, 2015

16. Leake D, Gunnlaugsson C, Urban J, Marentette L: Reconstruction after resection of sphenoid wing meningiomas. Arch Facial Plast Surg 7:99-103, 2005

17. MacCarty CS: Meningiomas of the sphenoidal ridge. J Neurosurg 36:114-120, 1972

18. Maroon JC, Kennerdell JS, Vidovich DV, Abla A, Sternau L: Recurrent spheno-orbital meningioma. J Neurosurg 80:202208, 1994

19. McDermott MW, Durity FA, Rootman J, Woodhurst WB: Combined frontotemporal-orbitozygomatic approach for tumors of the sphenoid wing and orbit. Neurosurgery 26:107116,1990

20. Mirone G, Chibbaro S, Schiabello L, Tola S, George B: En plaque sphenoid wing meningiomas: recurrence factors and surgical strategy in a series of 71 patients. Neurosurgery 65 (6 Suppl):100-109, 2009

21. Oya S, Sade B, Lee JH: Sphenoorbital meningioma: surgical technique and outcome. J Neurosurg 114:1241-1249, 2011

22. Pettersson-Segerlind J, Orrego A, Lönn S, Mathiesen T: Long-term 25-year follow-up of surgically treated parasagittal meningiomas. World Neurosurg 76:564-571, 2011

23. Pompili A, Derome PJ, Visot A, Guiot G: Hyperostosing meningiomas of the sphenoid ridge-clinical features, surgical therapy, and long-term observations: review of 49 cases. Surg Neurol 17:411-416, 1982

24. Ringel F, Cedzich C, Schramm J: Microsurgical technique and results of a series of 63 spheno-orbital meningiomas. Neurosurgery 60 (4 Suppl 2):214-222, 2007

25. Rogers L, Barani I, Chamberlain M, Kaley TJ, McDermott M, Raizer J, et al: Meningiomas: knowledge base, treatment outcomes, and uncertainties. A RANO review. J Neurosurg 122:4-23, 2015

26. Rogers L, Zhang P, Vogelbaum MA, Perry A, Ashby LS, Modi JM, et al: Intermediate-risk meningioma: initial outcomes from NRG Oncology RTOG 0539. J Neurosurg 129:35-47, 2018

27. Saeed P, van Furth WR, Tanck M, Freling N, van der Sprenkel JW, Stalpers LJ, et al: Surgical treatment of sphenoorbital meningiomas. Br J Ophthalmol 95:996-1000, 2011

28. Saeed P, van Furth WR, Tanck M, Kooremans F, Freling N, Streekstra GI, et al: Natural history of spheno-orbital meningiomas. Acta Neurochir (Wien) 153:395-402, 2011

29. Sandalcioglu IE, Gasser T, Mohr C, Stolke D, Wiedemayer $\mathrm{H}$ : Spheno-orbital meningiomas: interdisciplinary surgical approach, resectability and long-term results. J Craniomaxillofac Surg 33:260-266, 2005

30. Scarone P, Leclerq D, Héran F, Robert G: Long-term results with exophthalmos in a surgical series of 30 sphenoorbital meningiomas. Clinical article. J Neurosurg 111:1069-1077, 2009

31. Schick U, Bleyen J, Bani A, Hassler W: Management of meningiomas en plaque of the sphenoid wing. J Neurosurg 104:208-214, 2006

32. Shrivastava RK, Segal S, Camins MB, Sen C, Post KD: Harvey Cushing's Meningiomas text and the historical origin of resectability criteria for the anterior one third of the superior sagittal sinus. J Neurosurg 99:787-791, 2003

33. Shrivastava RK, Sen C, Costantino PD, Della Rocca R: Sphenoorbital meningiomas: surgical limitations and lessons learned in their long-term management. J Neurosurg 103:491-497, 2005

34. Talacchi A, De Carlo A, D’Agostino A, Nocini P: Surgical management of ocular symptoms in spheno-orbital meningiomas. Is orbital reconstruction really necessary? Neurosurg $\operatorname{Rev} 37: 301-310,2014$

35. Terrier LM, Bernard F, Fournier HD, Morandi X, Velut S, 
Hénaux PL, et al: Spheno-orbital meningiomas surgery: multicenter management study for complex extensive tumors. World Neurosurg 112:e145-e156, 2018

36. Weber DC, Ares C, Villa S, Peerdeman SM, Renard L, Baumert BG, et al: Adjuvant postoperative high-dose radiotherapy for atypical and malignant meningioma: a phase-II parallel non-randomized and observation study (EORTC 22042-26042). Radiother Oncol 128:260-265, 2018

\section{Disclosures}

The authors report no conflict of interest concerning the materials or methods used in this study or the findings specified in this paper.

\section{Author Contributions}

Conception and design: Magill, Dalle Ore, Idowu, Vagefi, McDermott. Acquisition of data: all authors. Analysis and interpretation of data: Magill, Dalle Ore, Idowu. Drafting the article: Magill, Dalle Ore, Vagefi, McDermott. Critically revising the article: Magill, Dalle Ore, Rodriguez Rubio, Vagefi, McDermott. Reviewed submitted version of manuscript: Magill, Dalle Ore,
Rodriguez Rubio, Shahin, McDermott. Approved the final version of the manuscript on behalf of all authors: Magill. Statistical analysis: Magill, Dalle Ore. Study supervision: Magill, Aghi, Theodosopoulos, Villanueva-Meyer, Vagefi, McDermott.

\section{Supplemental Information}

Previous Presentations

Earlier versions of this project were presented at the International Meningioma Society Annual Meeting in Prague, Czech Republic, in August 2018 and at the Western Neurosurgical Society Annual Meeting in Scottsdale, AZ, in November 2019.

\section{Correspondence}

Stephen T. Magill: University of California, San Francisco, CA. stephen.magill@ucsf.edu. 